\title{
Capsaicin enhances the antitumor activity of sorafenib in hepatocellular carcinoma cells and mouse xenograft tumors through increased ERK signaling
}

\author{
Su-shan ZHANG", Yu-hao NI", Chen-ru ZHAO, Zhen QIAO, Hong-xia YU, Lu-yao WANG, Jin-yan SUN, Chen DU, Jia-hao \\ ZHANG, Li-ying DONG, KeWei WANG * , Jian-jun GAO*
}

Department of Pharmacology, School of Pharmacy, Qingdao University, Qingdao 266021, China

\begin{abstract}
Sorafenib, a small inhibitor of tyrosine protein kinases, is currently the standard chemotherapy drug for the treatment of advanced hepatocellular carcinoma (HCC). Although sorafenib improves the survival of HCC patients, its efficacy is not optimal and requires further improvement. Capsaicin, the major active component of chili peppers from the genus Capsicum, is not only the agonist of TRPV1 channel, but also displays antitumor activity and enhances the sensitivity of cancer cells to cytotoxic drugs. In this study, we investigated the antitumor effects of combined sorafenib and capsaicin on HCC cells in vitro and xenograft tumors. Treatment with capsaicin alone dose-dependently inhibited the proliferation of the HCC cell lines PLC/PRF/7, HuH7 and HepG2 with IC $5_{50}$ values of 137, 108 and $140.7 \mu \mathrm{mol} / \mathrm{L}$, respectively. No obvious expression of TRPV1 channel was detected in the 3 HCC cell lines and TRPV1 channel blockers did not alleviate the cytotoxicity of capsaicin. By contrast, combining capsaicin and sorafenib significantly enhanced the suppression on cell proliferation, achieving a high-level synergistic effect (inhibition rates over $50 \%$ ) and promoting HCC cell apoptosis. In nude mice with PLC/PRF/5 xenografts, combined administration of capsaicin and sorafenib significantly enhanced the suppression on tumor growth without apparent gross toxicity compared to either agent alone. Mechanistically, capsaicin (10-200 $\mu \mathrm{mol} / \mathrm{L})$ dosedependently increased the levels of phosphorylated ERK ( $p$-ERK) in PLC/PRF/5 cells, thus leading to enhanced sorafenib sensitivity and a synergistic suppression on the tumor cells. Taken together, our results suggest that capsaicin-increased phosphorylation of ERK contributes to the enhanced antitumor activity of sorafenib, and capsaicin may be useful in improving the efficacy of sorafenib for the treatment of HCC.
\end{abstract}

Keywords: hepatocellular carcinoma; capsaicin; sorafenib; synergy; ERK; TRPV1

Acta Pharmacologica Sinica (2018) 39: 438-448; doi: 10.1038/aps.2017.156; published online 30 Nov 2017

\section{Introduction}

Hepatocellular carcinoma (HCC) remains a severe health threat worldwide ${ }^{[1-3]}$. Many patients are not diagnosed until advanced stages and require systemic chemotherapy ${ }^{[4-6]}$. Sorafenib, a small inhibitor of tyrosine protein kinases, is currently the only and standardized systemic chemotherapy drug for the treatment of advanced $\mathrm{HCC}^{[7-9]}$. Sorafenib is known to target non-receptor tyrosine kinase RAF and several receptor tyrosine kinases, including vascular endothelial growth factor receptor (VEGFR) and platelet-derived growth

\footnotetext{
\#These authors contributed equally to this work.

* To whom correspondence should be addressed.

E-mail wangkw@qdu.edu.cn (KeWei WANG); gaojj@qdu.edu.cn (Jian-jun GAO)

Received 2017-02-21 Accepted 2017-07-27
}

factor receptor (PDGFR) ${ }^{[10]}$. Clinical trials demonstrate that sorafenib prolongs the overall survival of HCC patients with a median of 2.8 months $^{[7,8]}$. Although sorafenib improves the overall survival, its efficacy remains suboptimal and requires further improvement. Therefore, identifying agents that can enhance the antitumor activity of sorafenib will be beneficial for the treatment of HCC patients.

Capsaicin (trans-8-methyl-N-vanillyl-6-nonenamide), the major active component of chili peppers from the genus Capsicum, has been widely used as a food additive and topical drug ${ }^{[11,12]}$. Capsaicin has attracted considerable attention because of its chemoprotective properties against certain carcinogens and mutagens ${ }^{[13]}$. In addition, capsaicin has been reported to induce cell apoptosis in several tumor types, such as myeloid leukemia, colon cancer, and HCC ${ }^{[14-17]}$. The 
mechanisms whereby capsaicin induces cancer cell apoptosis were reported to be either dependent or independent on the transient receptor potential vanilloid 1 (TRPV1) channel that serves as a direct target of capsaicin ${ }^{[18,19]}$. The antitumor effect of capsaicin has been reported to correlate with its ability to elevate the level of reactive oxygen species (ROS), thus interfering with cellular signaling pathways involved in cell proliferation and apoptosis ${ }^{[19,20]}$. Capsaicin was shown to inhibit STAT3 phosphorylation while activating the RAF/ MEK/ERK pathway in multiple myeloma and prostate cancer cells $^{[20,21]}$. Because activation of the RAF/MEK/ERK pathway was positively correlated with the sensitivity of HCC cells to sorafenib $^{[22,23]}$, we therefore investigated the antitumor activity of combining capsaicin and sorafenib in HCC cells and xenograft tumors in the current study.

We herein report that capsaicin suppressed the proliferation of HCC cells via a TRPV1-independent mechanism. Combining capsaicin and sorafenib enhanced the suppression of cell proliferation and the induction of cell apoptosis in HCC cells. The Chou-Talalay analysis revealed a synergistic antitumor effect of the capsaicin and sorafenib combination. Our findings show that sorafenib-mediated inhibition of increased ERK signaling by capsaicin underlies the observed synergism, and capsaicin may be a useful adjunct therapeutic approach for improving the efficacy of sorafenib.

\section{Materials and methods Agents}

Sorafenib and capsaicin were purchased from Selleckchem (Huston, USA) and Tocris Bioscience (Bristol, UK), respectively. For the in vitro assays, sorafenib and capsaicin were first dissolved in dimethyl sulfoxide (DMSO; Sigma-Aldrich) before further diluted with culture medium to the desired concentrations. For the in vivo experiments, sorafenib was formulated as previously described ${ }^{[24]}$ by dissolving in Cremophor EL/ethanol (50:50) and further diluting with water to the final dosing solution. Capsaicin was dissolved in ethanol before being diluted with normal saline to the desired concentration, with a final ethanol concentration of $0.2 \%$ for in vivo studies.

\section{Cell lines and cell culture}

Three HCC cell lines, including PLC/PRF/5, HuH7, and HepG2, and one normal human liver cell line, HL-7702, were employed in the present study. PLC/PRF/5 and HuH7 were obtained from the China Cell Bank (Shanghai, China). HepG2 was obtained from the Cell Resource Center, Peking Union Medical College (Beijing, China). HL-7702 cells were obtained from the China Center for Type Culture Collection (Wuhan, China). PLC/PRF/5 cells were transiently transfected with human TRPV1 cDNA (the accession number: NM_080704.3) using Lipofectamine 2000 transfection reagent (Invitrogen), and the transfection efficiency was approximately $72 \%$. The TRPV1 cDNA insert was generated with primers 5'-ATCGATGAAGAAATGGAGCAGCA-3' (forward) and 5'-ACTGTCACTTCTCCCCGGAAGC-3' (reverse) using LA
Taq (TAKARA) and subcloned into Bgl II and Sal I restriction sites of the pIRES2-EGFP vector. The cancer cells were maintained in Minimum Essential Medium (MEM; Thermo Fisher Scientific) supplemented with $10 \%$ fetal bovine serum (FBS; PAN Biotech). HL-7702 cells were maintained in RPMI1640 medium (Thermo Fisher Scientific) supplemented with $20 \%$ FBS (PAN Biotech). All cells were cultured at $37^{\circ} \mathrm{C}$ in a humid atmosphere $\left(5 \% \mathrm{CO}_{2}, 95 \%\right.$ air $)$.

\section{Cell proliferation assay}

Cells $\left(5 \times 10^{3}\right.$ per well) seeded in 96-well plates were exposed to capsaicin, sorafenib, or their combination for $72 \mathrm{~h}$. Then, the medium was removed and the wells were washed with phosphate-buffered saline (PBS). The 3-(4,5-dimethylthiazol2-yl)-2,5-diphenyltetrazolium bromide (MTT) assay was performed by adding $20 \mu \mathrm{L}$ of MTT ( $5 \mathrm{mg} / \mathrm{mL}$, Sigma-Aldrich) to the cells for $4 \mathrm{~h}$. Light absorbance was measured at $490 \mathrm{~nm}$ on a microplate reader (TECON, Swissland).

\section{Cell apoptosis detection}

Cell apoptosis was detected by Hoechst 33258 dye and propidium iodide (PI) staining, enzyme-linked immunosorbent assay (ELISA), and caspase $3 / 7$ activation assay.

For Hoechst 33258 and PI staining as well as the caspase 3/7 activation assay, cells $\left(5 \times 10^{3}\right.$ per well) seeded in 96 -well plates were exposed to capsaicin, sorafenib, or their combination for $24 \mathrm{~h}$. After being washed with PBS, cells were stained with Hoechst $33258(1 \mu \mathrm{g} / \mathrm{mL})$ and PI $(1 \mu \mathrm{g} / \mathrm{mL})$ for $20 \mathrm{~min}$. The apoptotic cells were quantified by calculating the ratio of PI-positive cells to Hoechst 33258-positive cells. Caspase 3/7 activity was examined using CellEvent Caspase-3/7 Green Detection Reagent (ThermoFisher Scientific) according to the manufacturer's directions.

For the ELISA, cells $\left(1 \times 10^{4}\right.$ per well) seeded in 96-well plates were exposed to capsaicin, sorafenib, or their combination for $24 \mathrm{~h}$. Cell apoptosis was detected using the Cell Death Detection ELISA ${ }^{\text {PLUS }}$ kit (Roche) according to the manufacturer's instructions. Cells were lysed with 200 $\mu \mathrm{L}$ of lysis buffer at room temperature for $30 \mathrm{~min}$. Then, cell lysates were centrifuged at $200 \times g$ for $10 \mathrm{~min}$ at $4{ }^{\circ} \mathrm{C}$. Twenty microliters of the supernatant was transferred into a streptavidin-coated microwell plate, $80 \mu \mathrm{L}$ of the immunoreagent containing Anti-DNA-POD and Anti-histonebiotin was added to each well, and the plate was incubated at room temperature for $2 \mathrm{~h}$ with shaking. The wells were washed three times, $100 \mu \mathrm{L}$ ABTS was added, and the plate was incubated for 10-20 min before the absorbance was measured at $405 \mathrm{~nm}$.

\section{Western blotting}

Cell lysates were prepared using RIPA lysis buffer (AR0105; Boster, Wuhan, China) containing cocktail protease inhibitor (AR1182; Boster) and phosphatase inhibitor (AR1183; Boster). Protein samples were denatured at $95{ }^{\circ} \mathrm{C}$ for $5 \mathrm{~min}$, separated by SDS-PAGE and transferred to polyvinylidene fluoride (PVDF) membranes (Millipore). After blocking with 5\% milk 
for $1 \mathrm{~h}$, the membranes were incubated with primary antibodies against TRPV1 (1:2000; 22686-1-AP; Proteintech), p-ERK1/2 (1:1000; ab33258; Abcam), p-STAT3 (1:100 000; ab76315; Abcam), Bax (1:1000; 50599-2-lg; Proteintech), Bcl-2 (1:1000; 12789-1-AP; Proteintech) and $\beta$-actin (1:1000; Boster) overnight at $4{ }^{\circ} \mathrm{C}$. After extensive washing in Tris-buffered saline with Tween-20 (TBST) buffer, the membranes were incubated with horseradish peroxidase-conjugated secondary antibody for 1 $\mathrm{h}$ at room temperature. The signal was detected using an ECL Western blotting detection system (Millipore).

\section{In vivo suppression of tumor growth}

The in vivo efficacy of combining capsaicin and sorafenib was assessed using a PLC/PRF/5 xenograft mouse model. Athymic female mice (NU/NU), 4-6 weeks of age, were purchased from Beijing Vital River Laboratory Animal Technology Co, Ltd (Beijing, China). The animals were housed under pathogen-free conditions. The research protocol was in accordance with the institutional guidelines of the Animal Care and Use Committee.

Tumors were generated by harvesting PLC/PRF/ 5 cells from mid-log phase cultures using trypsin-EDTA (ThermoFisher Scientific). Cells were then pelleted and resuspended in PBS to a final cell count of $5 \times 10^{7} / \mathrm{mL}$. A volume of $0.2 \mathrm{~mL}$ of the cell suspension was injected sc in the right flank of each mouse. After $7 \mathrm{~d}$, when the tumor volume had reached approximately
$0.1 \mathrm{~cm}^{3}$, all the mice were divided into 4 groups $(n=7)$. In group 1, mice received an intratumor injection of $100 \mu \mathrm{L}$ normal saline containing $0.2 \%$ ethanol. In group 2 , mice received $50 \mathrm{mg} / \mathrm{kg}$ sorafenib (po). In group 3, mice received an intratumor injection of $100 \mu \mathrm{L}$ capsaicin solution $(200 \mu \mathrm{mol} / \mathrm{L})$. In group 4, mice received $50 \mathrm{mg} / \mathrm{kg}$ sorafenib (po) and an intratumor injection of $100 \mu \mathrm{L}$ capsaicin solution $(200 \mu \mathrm{mol} / \mathrm{L})$. Administration of normal saline, sorafenib, capsaicin, or their combination was performed daily for $21 \mathrm{~d}$. Tumor volume and body weight were measured every $4 \mathrm{~d}$ throughout the experiment.

\section{Statistical analysis}

The data are expressed as the mean $\pm S D$ for three different determinations. Statistical significance was analyzed by oneway analysis of variance (ANOVA) followed by Dunnett's multiple range tests. The value of $P<0.05$ was considered to be statistically significant. Statistical analysis was performed using the SPSS/Win 16.0 software (SPSS, Inc, Chicago, IL, USA).

\section{Results}

Inhibitory effect of capsaicin or sorafenib on tumor cell viability The effect of capsaicin or sorafenib on cell viability was determined using an MTT assay. Three HCC cell lines, PLC/PRF/5, HuH7, and HepG2, were treated with different
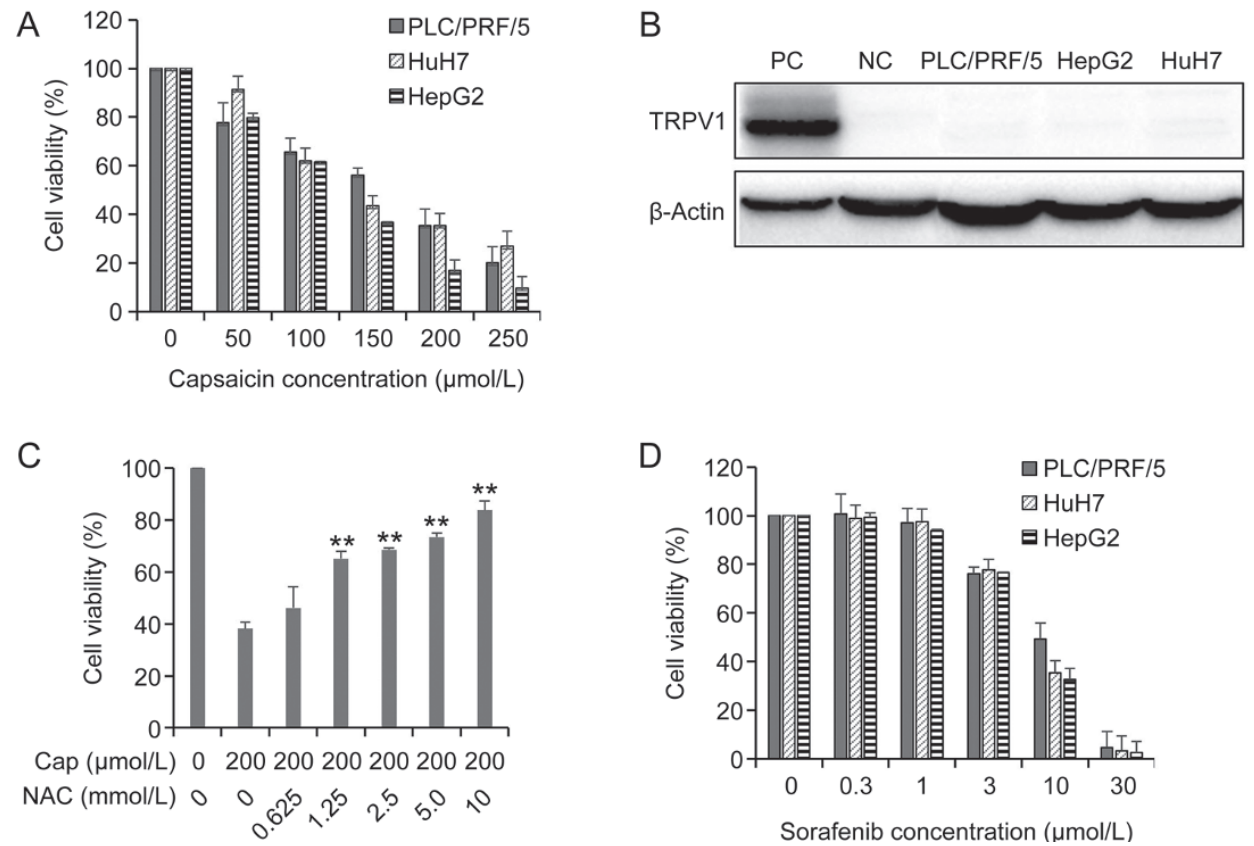

Figure 1. Inhibitory effect of capsaicin or sorafenib on tumor cell viability. (A) Capsaicin dose-dependently reduced the viability of the HCC cells PLC/ $\mathrm{PRF} / 5, \mathrm{HuH} 7$, and HepG2. Cells were incubated with different concentrations of capsaicin for $72 \mathrm{~h}$ before being subjected to an MTT assay. (B) TRPV1 expression was not detected in PLC/PRF/5, HuH7, or HepG2 cells. HEK293-TRPV1 cells (HEK293 cells transfected with a plasmid containing the trpv1 gene; positive control, PC), HEK293 cells (negative control, NC), and the three HCC cell lines were lysed and subjected to Western blotting assay. (C) $\mathrm{N}$-acetyl-L-cysteine (NAC) does-dependently alleviated the capsaicin-induced cytotoxicity in PLC/PRF/5 cells. Cells were exposed to both capsaicin and different concentrations of NAC for $72 \mathrm{~h}$ before being subjected to an MTT assay. Cap, capsaicin; NAC, N-acetyl-L-cysteine. ${ }^{* *} P<0.01$. (D) Sorafenib dose-dependently decreased the viability of PLC/PRF/5, HuH7, and HepG2 cells. Cells were incubated with different concentrations of sorafenib for 72 $h$ before being subjected to an MTT assay. 
concentrations of capsaicin $(0,50,100,150,200$, and 250 $\mu \mathrm{mol} / \mathrm{L})$ or sorafenib $(0,0.3,1,3,10$, and $30 \mu \mathrm{mol} / \mathrm{L})$ for $72 \mathrm{~h}$. As shown in Figure 1A, capsaicin inhibited cell proliferation in all three cell lines in a dose-dependent manner, with $\mathrm{IC}_{50}$ values of $137.0,108.0$, and $140.7 \mu \mathrm{mol} / \mathrm{L}$, respectively. As a well-known agonist of TRPV1, capsaicin activates the channel
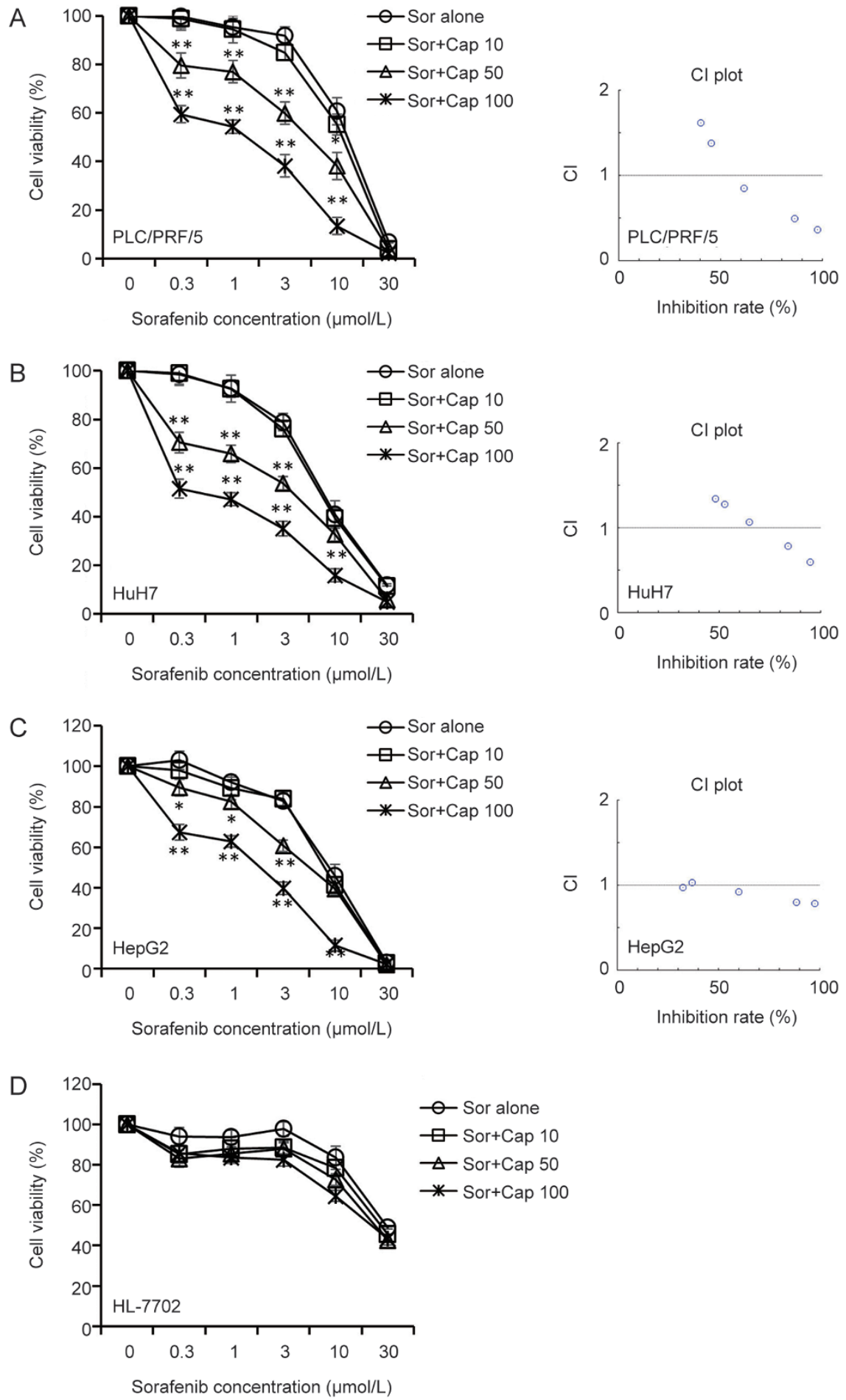

Figure 2. Synergistic inhibition of tumor cell viability by combined sorafenib and capsaicin. Capsaicin dose-dependently enhanced the cytotoxicity induced by sorafenib in the HCC cells PLC/PRF/5 (A, left panel), HuH7 (B, left panel), and HepG2 (C, left panel) and in normal human liver cells HL7702 (D). Cells were exposed to sorafenib alone or in combination with capsaicin at a fixed concentration (10, 50, or 100 $\mu \mathrm{mol} / \mathrm{L}$, respectively) for 72 $\mathrm{h}$ before being subjected to an MTT assay. Combination indexes (Cls) of sorafenib plus capsaicin (100 $\mu$ mol/L) were calculated using the CompuSyn software and were plotted against cell inhibition rates (right panel). Sor, sorafenib; Cap, capsaicin. ${ }^{*} P<0.05,{ }^{* *} P<0.01$. 
at a concentration of $1 \mu \mathrm{mol} / \mathrm{L}^{[25]}$. However, capsaicin at this concentration had no effect on the viability of the three cell lines used in this study, suggesting that inhibition of cell growth by capsaicin is independent of TRPV1 channel activation. To confirm this hypothesis, we examined TRPV1 expression in three cell lines and detected no obvious TRPV1 band (Figure 1B). In addition, we tested two different blockers, ruthenium red, a pan-inhibitor of TRPVs, and BAPTA-AM, a selective intracellular $\mathrm{Ca}^{2+}$ chelator, that did not alleviate the cytotoxicity of capsaicin (data not shown), confirming that the inhibitory effect of cell growth by capsaicin is not ascribed to TRPV1 activation and the subsequent $\mathrm{Ca}^{2+}$ influx. We further tested whether ectopic expression of TRPV1 in HCC cells had any impact on cell viability by comparing the effect of capsaicin on TRPV1-transfected PLC/PRF/5 cells (PLC/ PRF/5-TRPV1) with control vector-transfected PLC/PRF/5 cells (PLC/PRF/5-vector). The results showed that the $\mathrm{IC}_{50}$ values of capsaicin on either PLC/PRF/5-TRPV1 cells (174.3 $\mu \mathrm{mol} / \mathrm{L})$ or PLC/PRF/5-vector cells $(166.8 \mu \mathrm{mol} / \mathrm{L})$ were similar, indicating an insignificant role of TRPV1 in mediating the antitumor effect of capsaicin on HCC cells.

As the induction of ROS generation was reported to be associated with antitumor activity of capsaicin, we investigated whether $N$-acetyl-L-cysteine (NAC), a membranepenetrating antioxidant that reduces ROS levels, could alleviate the cytotoxic effect of capsaicin on cancer cells. Not surprisingly, NAC dose-dependently improved the viability of PLC/PRF/ 5 cells that were concurrently exposed to capsaicin (Figure 1C), indicating that ROS generation plays an important role in capsaicin-induced cytotoxicity.

Sorafenib is known to repress HCC cell growth, and we also determined the effect of sorafenib on cell growth. As shown in Figure 1D, sorafenib suppressed the growth of PLC/ PRF/5, HuH7, and HepG2 cells in a dose-dependent manner, exhibiting significant inhibition of cell viability in all three HCC cell lines at concentrations above $3 \mu \mathrm{mol} / \mathrm{L}$ with $\mathrm{IC}_{50}$ values of 7.6, 6.4, and $5.9 \mu \mathrm{mol} / \mathrm{L}$, respectively (Figure 1D).

\section{Synergistic inhibition of tumor cell viability by combined sorafenib and capsaicin}

To investigate the antitumor effect of combined sorafenib and capsaicin, cells were treated with different concentrations of sorafenib in the presence or absence of capsaicin for $72 \mathrm{~h}$ and the cell viability was determined by an MTT assay. As shown in Figure 2A, sorafenib at the concentration range of $0-30 \mu \mathrm{mol} / \mathrm{L}$ resulted in a leftward shift of cell growth inhibition of PLC/PRF/ 5 cells in the presence of 50 or 100 $\mu \mathrm{mol} / \mathrm{L}$ capsaicin compared with sorafenib alone. To explore the interactive effect of sorafenib and capsaicin, we calculated the combination index $(\mathrm{CI})$ values of sorafenib plus capsaicin $(100 \mu \mathrm{mol} / \mathrm{L})$ using the CompuSyn software and determined the interaction types according to the Chou-Talalay Method (Figure 2, right panels). The analysis revealed that combining sorafenib and capsaicin gave rise to a synergistic effect when sorafenib was used at relatively high concentrations $(3,10$, and $30 \mu \mathrm{mol} / \mathrm{L})$, and a moderate or slight antagonistic effect when sorafenib was used at low concentrations $(0.3$ or $1.0 \mu \mathrm{mol} / \mathrm{L}$; Figure 2A). A similar effect of sorafenib plus capsaicin was also observed in other two HCC cells lines, HuH7 and HepG2 (Figures 2B and 2C). A synergistic effect on cell growth inhibition was observed when capsaicin was combined with sorafenib at relatively high concentrations, while a slight antagonistic or nearly additive effect was observed when capsaicin was combined with relatively low concentrations of sorafenib. From the perspective of inhibition rate, our results indicate that capsaicin in combination with sorafenib is prone to a synergistic effect on HCC cells at high inhibition rates (approximately over 50\%; Figure 2, right panel).

To determine the side effects of sorafenib, capsaicin, and the drug combination on normal cells, we examined their cytotoxic effects on the normal human liver cell line HL-7702. The results showed that sorafenib alone and capsaicin alone suppressed the growth of HL-7702 cells in a dose-dependent manner, with $\mathrm{IC}_{50}$ values of $28.3 \mu \mathrm{mol} / \mathrm{L}$ and $317.6 \mu \mathrm{mol} / \mathrm{L}$, respectively, which are significantly higher than those values in HCC cells, suggesting that both sorafenib and capsaicin exhibited a lower cytotoxicity to normal liver cells (Figure 2D). The combination of capsaicin and sorafenib exhibited an enhanced cytotoxic effect on the normal cells. However, the increased cytotoxicity in normal cells was lower than that in HCC cells.

\section{Combining sorafenib and capsaicin enhances apoptotic cell death}

It has been reported that sorafenib alone and capsaicin alone are capable of inducing apoptosis in cancer cells ${ }^{[10,17]}$. We examined the apoptosis induced by the capsaicin and sorafenib combination in the HCC cell line PLC/PRF/5. The cells were exposed to sorafenib ( 5 and $10 \mu \mathrm{mol} / \mathrm{L}$ ) in the presence or absence of capsaicin $(100 \mu \mathrm{mol} / \mathrm{L})$ for $24 \mathrm{~h}$ and subsequently stained with Hoechst 33258 and PI. As shown in Figure $3 \mathrm{~A}$ and $3 \mathrm{~B}$, sorafenib alone at 5 or $10 \mu \mathrm{mol} / \mathrm{L}$ increased the percentage of PI-positive cells from $0.41 \%$ to $5.6 \%$ and $9.8 \%$, respectively. Capsaicin alone at $100 \mu \mathrm{mol} / \mathrm{L}$ increased the percentage of PI-positive cells by $3.8 \%$. Co-treatment of PLC/PRF/ 5 cells with sorafenib and $100 \mu \mathrm{mol} / \mathrm{L}$ capsaicin significantly increased sorafenib-induced apoptosis as measured by an increase of $13.37 \%$ (capsaicin plus $5 \mu \mathrm{mol} / \mathrm{L}$ sorafenib) and $30.58 \%$ (capsaicin plus $10 \mu \mathrm{mol} / \mathrm{L}$ sorafenib) in PI-positive cells. Using the Cell Death Detection ELISA, we confirmed that the combination of capsaicin and sorafenib showed an enhanced effect on cell apoptosis in PLC/PRF/5 cells (Figure 3C).

Caspases, a family of cysteine acid proteases, are central regulators of apoptosis. Active caspase 3/7, a hallmark of apoptosis, was detected in PLC/PRF/ 5 cells using a substrate reagent that emits green fluorescence after cleavage by active caspase $3 / 7$ (Figure $4 \mathrm{~A}$ ). The percentages of caspase 3/7-positive cells after exposure to the combination of sorafenib and capsaicin were increased by $8.0 \%$ and $27.0 \%$, compared to an increase of only $3.6 \%$ and $9.7 \%$ for the treatment of 5 and $10 \mu \mathrm{mol} / \mathrm{L}$ of sorafenib alone, respectively 

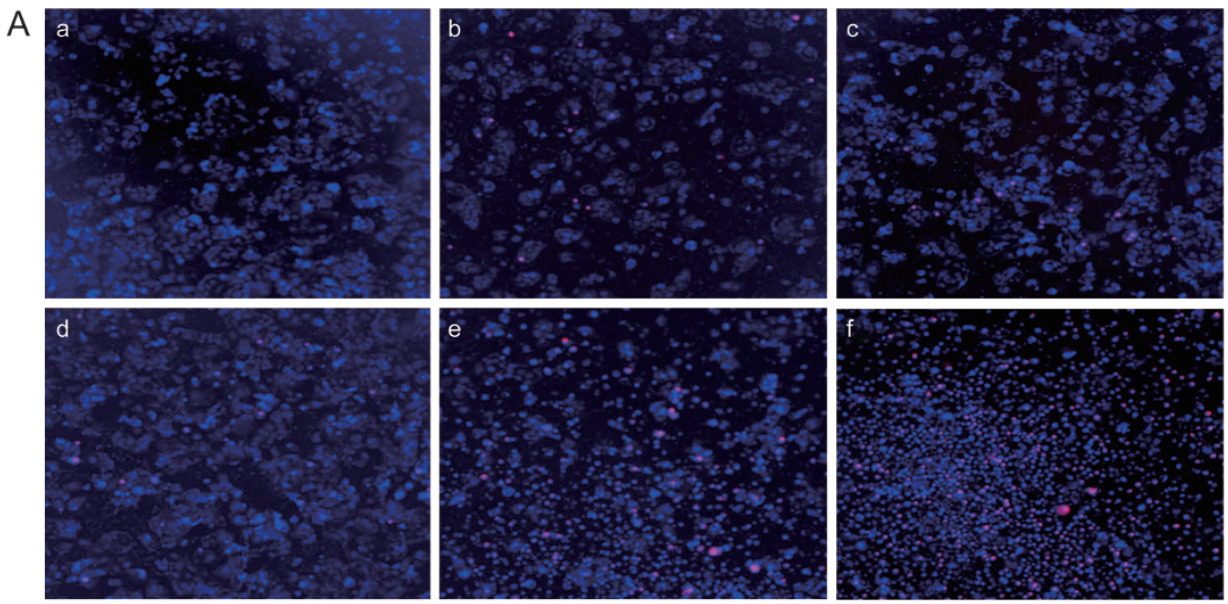

B

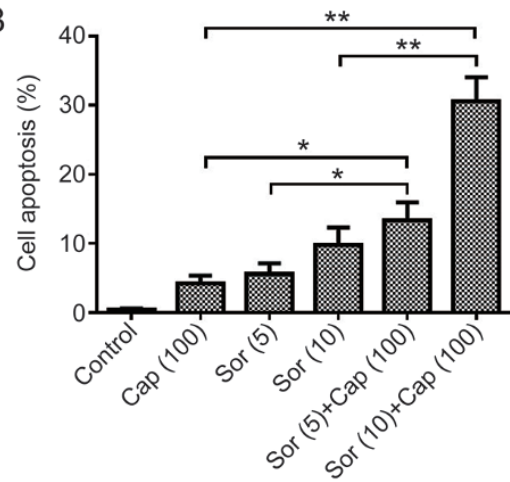

C

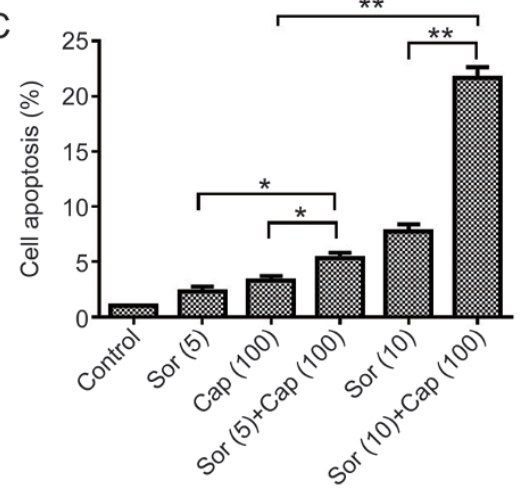

Figure 3. Increased apoptotic cell death induced by combined sorafenib and capsaicin. PLC/PRF/5 cells were exposed to sorafenib, capsaicin, or their combination for $24 \mathrm{~h}$. (A and B) Cells were stained with Hoechst 33258 and PI; the apoptotic cells were quantified by calculating the ratio of PI-positive cells to Hoechst 33258-positive cells. a, control; b, $5 \mu \mathrm{mol} / \mathrm{L}$ sorafenib; c, $10 \mu \mathrm{mol} / \mathrm{L}$ sorafenib; d, $100 \mu \mathrm{mol} / \mathrm{L}$ capsaicin; e, $5 \mu \mathrm{mol} / \mathrm{L}$ sorafenib plus $100 \mu \mathrm{mol} / \mathrm{L}$ capsaicin; f, $10 \mu \mathrm{mol} / \mathrm{L}$ sorafenib plus $100 \mu \mathrm{mol} / \mathrm{L}$ capsaicin. (C) Cell apoptosis was detected using a Cell Death Detection ELISA kit. Cap, capsaicin; Sor, sorafenib. ${ }^{*} P<0.05,{ }^{* *} P<0.01$.

(Figure 4B). These results indicated that capsaicin and sorafenib co-treatment was more efficient in activating caspase $3 / 7$ in HCC cells. Furthermore, we examined the effect of the capsaicin and sorafenib combination treatment on Bax and Bcl-2 intrinsic apoptotic markers using Western blot. The results demonstrated that capsaicin and sorafenib co-treatment increased Bax expression and decreased Bcl-2 expression in PLC/PRF/ 5 cells (Figure 4C). The Bax/Bcl-2 ratio was significantly increased in cells exposed to the drug combination compared to those exposed to capsaicin alone or sorafenib alone (Figure $4 \mathrm{C}$ ). These results suggested that a mitochondria-dependent pathway might be involved in cell apoptosis induced by the combination treatment.

Increased antitumor sensitivity of sorafenib by capsaicin through the enhancement of $\mathrm{p}$-ERK and reduction of p-STAT3 signaling To explore the mechanism underlying the synergistic effect of combined capsaicin and sorafenib, we examined the signal transduction of ERK and STAT3 that are critically involved in the effect of sorafenib. Sorafenib has been shown to inhibit the RAF/MEK/ERK pathway, and a higher level of phosphorylated ERK (p-ERK) was correlated with better survival rates in HCC patients receiving sorafenib treatment ${ }^{[23]}$, and STAT3 was reported to be a major kinase-independent target of sorafenib ${ }^{[26]}$. As shown in Figure 5A, sorafenib treatment decreased the expression of p-ERK and phosphorylated STAT3 (p-STAT3) in PLC/PRF/ 5 cells in a dose-dependent manner. Interestingly, capsaicin treatment caused a decrease in p-STAT3 and an increase in p-ERK1/2 in a dose-dependent manner (Figure 5B), implying that enhanced p-ERK1/2 expression induced by capsaicin is likely involved in the synergistic effect observed in the combination.

To further confirm the role of capsaicin in ERK phosphorylation, we examined the impact of the combined treatment on p-ERK1/2 and p-STAT3 signaling. As shown in Figure $5 \mathrm{C}$, capsaicin alone at a fixed concentration of $100 \mu \mathrm{mol} / \mathrm{L}$ significantly upregulated the level of p-ERK1/2, but this effect was abrogated by sorafenib in a concentration-dependent manner. We also tested the effect of capsaicin and sorafenib on p-STAT3, and found that the combined treatment significantly reduced the levels of p-STAT3 at three different concentrations $(2.5,5$, and $10 \mu \mathrm{mol} / \mathrm{L})$ of sorafenib (Figure 5C). The ERK and STAT pathways are widely reported oncogenic pathways in $\mathrm{HCC}$, and their activation may protect 

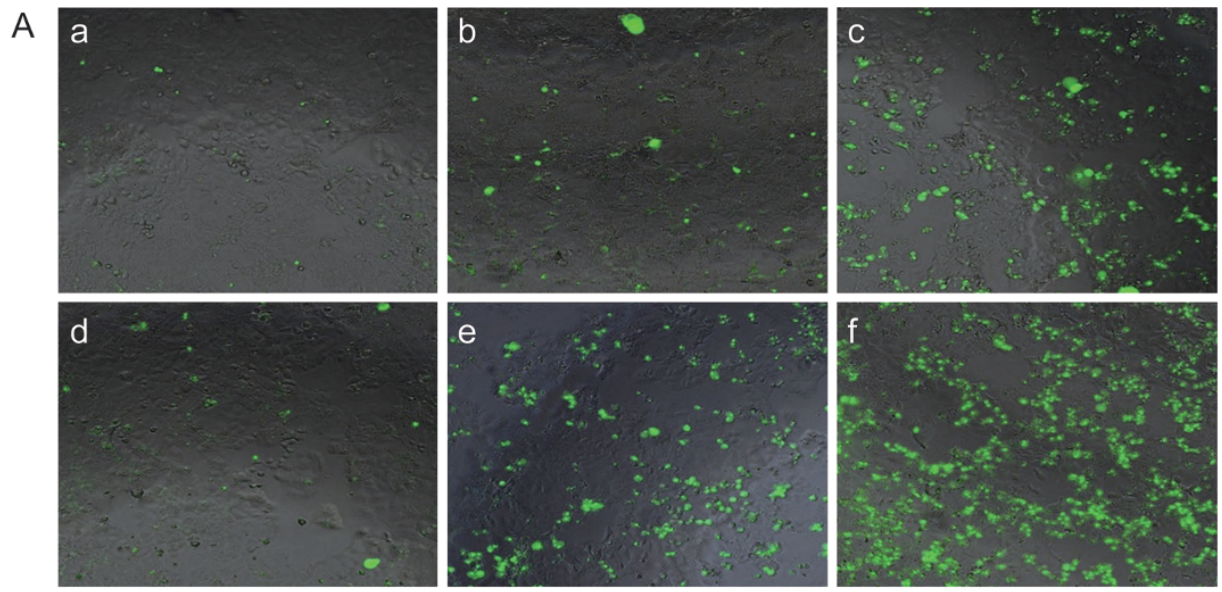

B

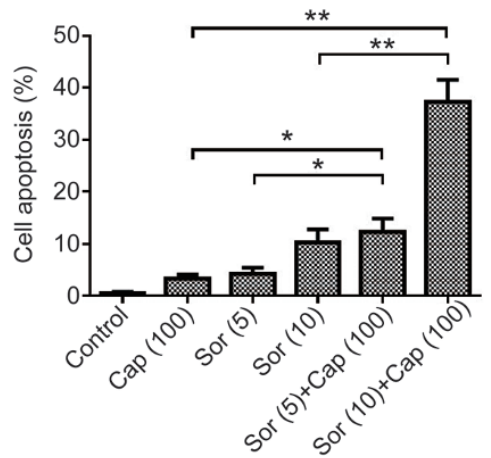

C

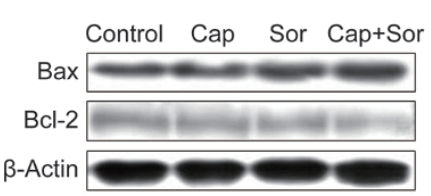

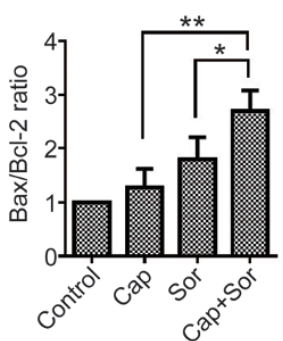

Figure 4. Expression of apoptosis-related proteins in HCC cells treated with combined sorafenib and capsaicin. PLC/PRF/5 cells were exposed to sorafenib, capsaicin, or their combination for $24 \mathrm{~h}$. (A and B) Cells were incubated with the CellEvent Caspase-3/7 Green Detection Reagent; apoptotic cells were quantified by calculating the ratio of the active caspase 3/7-positive cells to all cells in the well. a, control; b, $5 \mu \mathrm{mol} / \mathrm{L}$ sorafenib; c, $10 \mu \mathrm{mol} / \mathrm{L}$ sorafenib; d, $100 \mu \mathrm{mol} / \mathrm{L}$ capsaicin; e, $5 \mu \mathrm{mol} / \mathrm{L}$ sorafenib plus $100 \mu \mathrm{mol} / \mathrm{L}$ capsaicin; f, $10 \mu \mathrm{mol} / \mathrm{L}$ sorafenib plus $100 \mu \mathrm{mol} / \mathrm{L}$ capsaicin. (C) Bax and Bcl-2 expression levels were detected by Western blot and their ratios were quantified. Cap, capsaicin; Sor, sorafenib. ${ }^{*} P<0.05,{ }^{* *} P<0.01$.

cells from apoptosis. We speculate that downregulation of p-STAT may promote cell apoptosis while upregulation of p-ERK causes cell survival for maintaining a self-balance. To verify this hypothesis, we examined the effect of capsaicin in combination with PD032590, a selective inhibitor of ERK phosphorylation. The results showed that this combination also achieved synergism in suppressing the growth of PLC/ PRF/ 5 cells, suggesting that blocking the ERK pathway may enhance the antitumor effect of capsaicin (Figure 5D). Collectively, these results suggest that the activation of ERK by capsaicin, in contrast to the attenuation of ERK activation by sorafenib, and the co-suppression of STAT3 by the two agents are likely responsible for the synergistic inhibitory effect of the combined treatment on HCC cell growth.

\section{Enhanced inhibition of xenograft tumor growth in nude mice by} combining capsaicin and sorafenib

To assess the in vivo efficacy of sorafenib plus capsaicin, a PLC/PRF/ 5 tumor xenograft model was generated. Mice were randomly assigned to four groups: control, sorafenib, capsaicin, and the combination of sorafenib plus capsaicin. Oral administration of sorafenib at $50 \mathrm{mg} / \mathrm{kg}$ or intratumor injection of $100 \mu \mathrm{L}$ capsaicin at $200 \mu \mathrm{mol} / \mathrm{L}$ for $21 \mathrm{~d}$ resulted in a significant tumor growth inhibition (TGI) of approximately $58.9 \%$ and $52.2 \%$, respectively, compared with the vehicle control group (Figure 6A). In contrast, combining sorafenib $(50 \mathrm{mg} / \mathrm{kg}, p o$ ) with capsaicin (intratumor injection of 200 $\mu \mathrm{mol} / \mathrm{L}$ in $100 \mu \mathrm{L}$ ) gave rise to a greater suppression of tumor growth with a TGI of approximately $83.3 \%$ (Figure $6 \mathrm{~A})$. As an additional control, we also monitored the body weights of nude mice throughout the experimental period (Figure 6B). There was a slight weight loss observed in the sorafenib-treated, capsaicin-treated, and combination-treated groups. However, if the tumor weight was deducted, no significant difference in net body weight was found between the treatment groups compared with the vehicle control group at the end of experiment, suggesting that the slight weight loss was associated with tumor growth inhibition, and the sorafenib and capsaicin combination caused no apparent gross toxicity in the nude mice.

\section{Discussion}

The purpose of this study was to investigate the synergistic antitumor activity of sorafenib in combination with capsaicin. Our findings show that the combination of sorafenib and capsaicin synergistically repressed cell proliferation and induced 
A

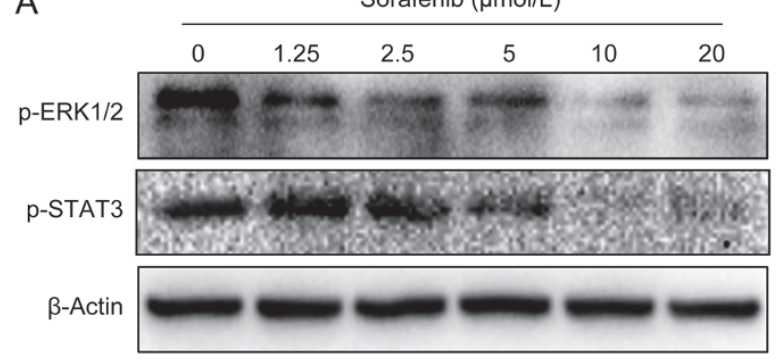

B

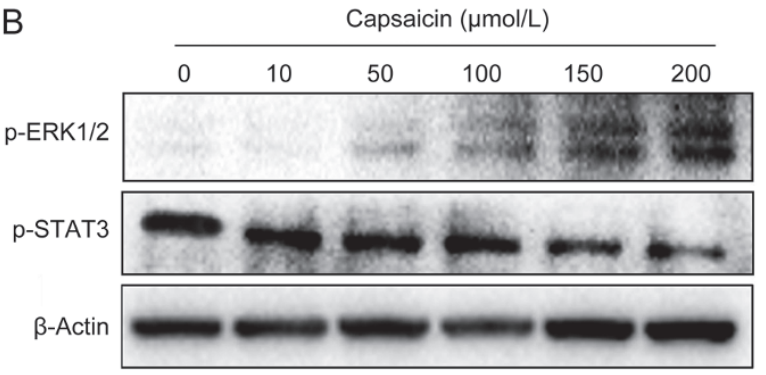

C

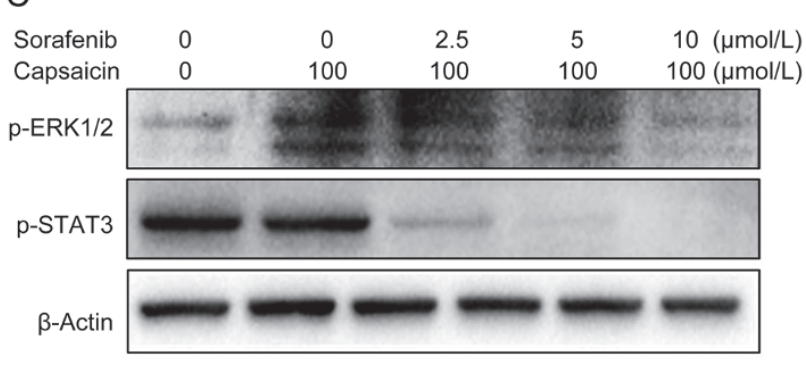

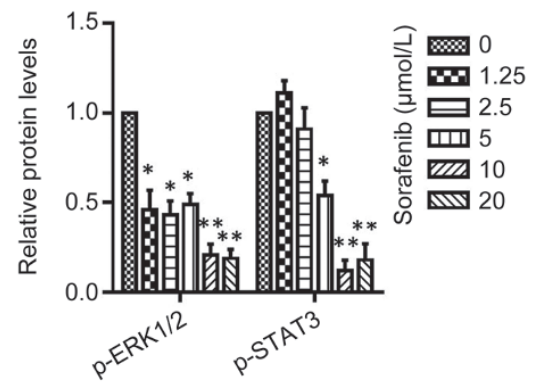
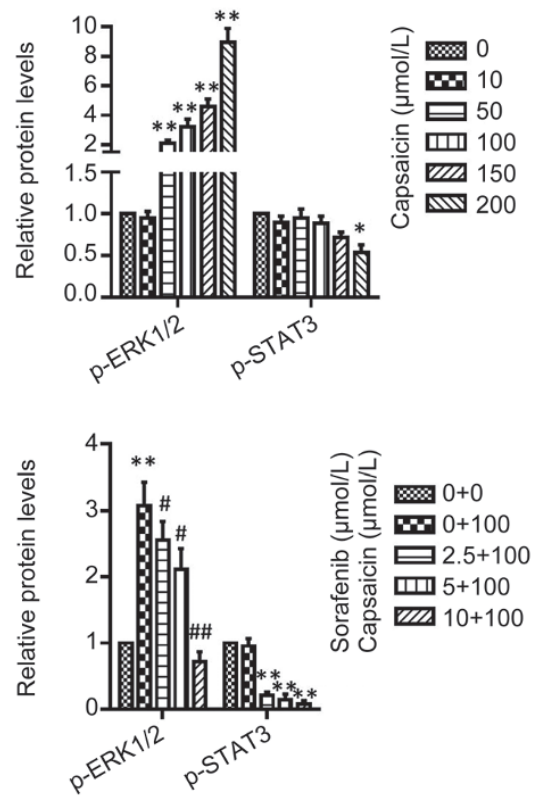

\section{D}

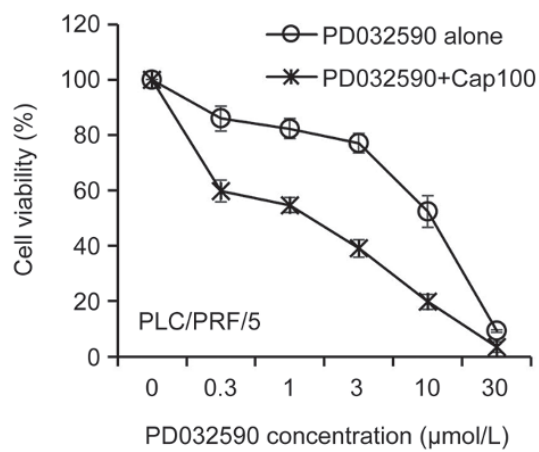

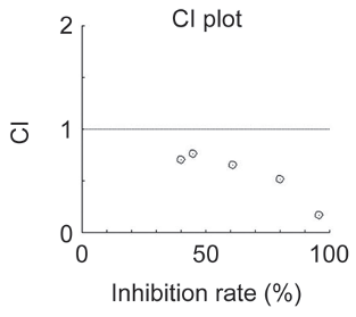

Inhibition rate $(\%)$

Figure 5. Increased antitumor sensitivity of sorafenib by capsaicin through the enhancement of $p$-ERK1/2 and the reduction of p-STAT3 levels. (AC) PLC/PRF/5 cells were incubated with sorafenib, capsaicin, or their combination for $24 \mathrm{~h}$ before being subjected to Western blot analysis. (A) Sorafenib decreased the levels of $\mathrm{p}$-ERK1/2 and p-STAT3 in a dose-dependent manner. ${ }^{*} P<0.05,{ }^{* *} P<0.01$ vs control. (B) Capsaicin increased the level of $p$-ERK1/2 and decreased the level of $p$-STAT3 in a dose-dependent manner. ${ }^{*} P<0.05,{ }^{* *} P<0.01$ vs control. (C) Capsaicin increased the level of p-ERK1/2, which was dose-dependently reduced by sorafenib; the combination treatment obviously decreased the level of $p$-STAT3. ${ }^{* *} P<0.01$ vs control. ${ }^{\#} P<0.05$, ${ }^{\# \#} P<0.01$ vs cells exposed to capsaicin alone. (D) $P L C / P R F / 5$ cells were exposed to PD032590 alone or in combination with capsaicin at a fixed concentration $(100 \mu \mathrm{mol} / \mathrm{L})$ for $72 \mathrm{~h}$ before being subjected to an MTT assay. Combination indexes (Cls) of PD032590 plus capsaicin were calculated using the CompuSyn software and were plotted against cell inhibition rates (right panel).

cell apoptosis in HCC cells. Capsaicin moderately inhibited the phosphorylation of STAT3, an oncogenic signaling molecule, and increased the level of p-ERK, which was abrogated by sorafenib in a concentration-dependent manner. We speculate that capsaicin renders cancer cells dependent on ERK sig- naling, thus leading to the increase in sorafenib sensitivity.

The combination of antitumor drug sorafenib and agents with alternated mechanisms of action may unfold new therapeutic options that can increase the survival of advanced HCC patients. Capsaicin exerts its physiologic function, ie, burning 

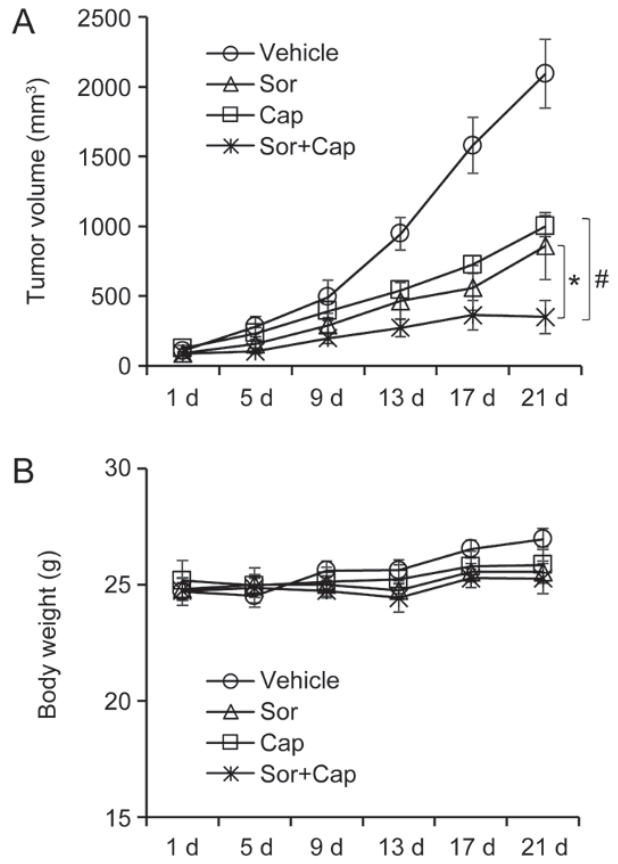

Figure 6. Enhanced inhibition of xenograft tumor growth in nude mice by combining sorafenib and capsaicin. Nude mice with PLC/PRF/5 xenografts received normal saline (intratumor injection, $100 \mu \mathrm{L}$ ), sorafenib (p.o., $50 \mathrm{mg} / \mathrm{kg}$ ), capsaicin (intratumor injection, $200 \mu \mathrm{mol} / \mathrm{L}$ or $100 \mu \mathrm{L}$ ), and the drug combination for $21 \mathrm{~d}$. Tumor volume (A) and body weight (B) were measured every 4 days. Mice were euthanized at the end of the experiment. Cap, capsaicin; Sor, sorafenib. ${ }^{*} P<0.05$ vs sorafenib; ${ }^{\#} P<0.05$ vs capsaicin.

sensation, in sensory neurons by binding to TRPV1, inducing $\mathrm{Ca}^{2+}$ influx, and generating action potential ${ }^{[27]}$. Our findings support the view that the antitumor effect of capsaicin is less likely involved in directly targeting the TRPV1 channel, although Yang et al reported that capsaicin mediated cell death in bladder cancer T24 cells through activating TRPV1 and inducing $\mathrm{Ca}^{2+}$ entry-dependent ROS production ${ }^{[28]}$. Several studies provided evidence supporting that the antitumor effect of capsaicin is not correlated with TRPV1 in prostate cancer cells ${ }^{[29]}$, gastric cancer cells ${ }^{[30]}$, or human small cell lung cancer ${ }^{[31]}$. Studies by Mori et al revealed that capsaicininduced apoptosis in prostate cancer cells was correlated with ROS production; however, the direct target of capsaicin was unknown $^{[29]}$. It is worth noting that capsaicin usually inhibits cell proliferation or induces cell apoptosis at concentrations far higher than those that activate TRPV1 ${ }^{[22]}$, suggesting that other targets are likely involved in its antitumor activity. In our study, TRPV1 was not detected in HCC cells, which also suggests that the antitumor effect of capsaicin is not correlated to TRPV1.

Since inhibition of ROS by the reducing agent NAC significantly alleviates the cytotoxic effect of capsaicin, the production of ROS may be involved in the antitumor effect of capsaicin. It has been demonstrated that ROS influence cell viability from multiple aspects. In addition to their activity in damaging cellular proteins, lipids, and DNA thus leading to cell apoptosis, ROS are also implicated in cell signal transduction, which facilitates or inhibits cell proliferation ${ }^{[33-35]}$. We and other researchers demonstrate that capsaicin decreases the level of p-STAT3 and increases the level of p-ERK, which likely underlies the synergistic effect when capsaicin is used in combination with sorafenib. Consistent with our studies, Jiang et al reported that p-ERK expression is a determinant factor for synergistic antitumor activity in sorafenib-based combination therapy. They found that DE605, a c-Met inhibitor, activates the MEK/ERK pathway, which in turn is inhibited by sorafenib, resulting in a synergistic antitumor effect in HCC cells $^{[36]}$. In our study, the increased level of p-ERK by capsaicin is efficiently reduced by relatively high concentrations of sorafenib (Figure 5C), which may help explain why capsaicin plus high concentrations of sorafenib are prone to achieving a synergistic antitumor effect.

The pharmacological activity of capsaicin is dependent on several factors, including the dose, the route of administration, and most importantly, its concentration in target tissues ${ }^{[37]}$. Mice orally gavaged with capsaicin at $10 \mathrm{mg} / \mathrm{kg}$ had a peak blood concentration of $1.17 \mu \mathrm{g} / \mathrm{mL}(3.8 \mu \mathrm{mol} / \mathrm{L})$ capsaicin at 1 $\mathrm{h}$ post-feeding, which is obviously lower than concentrations required for its activity against HCC cells ${ }^{[37]}$. Consideration of oral dose escalation was abandoned in our study because the high dose $(50 \mathrm{mg} / \mathrm{kg})$ of capsaicin produced a severe burning sensation that was intolerable to mice. We finally adopted the approach of intratumor injection of capsaicin in the current study. We found that capsaicin alone via this route inhibited the growth of PLC/PRF/5 xenografts and significantly increased the antitumor efficacy of sorafenib without obvious irritation, suggesting that capsaicin can be potentially used in a local therapeutic modality, such as hepatic transcatheter arterial chemoembolization (TACE), for HCC treatment. Although oral administration of high-dose capsaicin is not practical due to its irritative effect on the gastrointestinal tract, its application via oral administration could be an option if capsaicin can be administered through an appropriate drug carrier. Strategies that can mask the burning sensation of capsaicin and achieve targeted delivery, such as microsphereand nanosphere-based delivery systems, may be utilized to overcome the side effect of capsaicin. With the above considerations, capsaicin may possess a therapeutic potential to be used alone or in combination with other anticancer drugs such as sorafenib for HCC treatment.

\section{Conclusion}

In conclusion, combining sorafenib and capsaicin exhibits an enhanced activity in suppressing cell proliferation and inducing cell apoptosis in HCC cells. This combination can achieve a high-level synergistic antitumor effect, which may facilitate its application in cancer management. Our findings suggest that capsaicin may be useful as an adjunct therapeutic approach for the improvement of sorafenib efficacy in suppression of HCC. 


\section{Acknowledgements}

This project was supported by grants from the National Natural Science Foundation of China (81573410 to KeWei WANG and 81503094 to Jian-jun GAO), the China Postdoctoral Science Foundation (2016 M600524), and the Qingdao Postdoctoral Applied Research Project (2016072; Qingdao University).

\section{Author contribution}

Jian-jun GAO and KeWei Wang designed the research; Sushan ZHANG, Yu-hao NI, Chen-ru ZHAO, Zhen QIAO, Hong-xia YU, Lu-yao WANG, Jin-yan SUN, Chen DU, Jia-hao ZHANG, and Li-ying DONG performed the research; Su-shan ZHANG and Jian-jun GAO analyzed the data; Jian-jun GAO and KeWei WANG wrote the paper; Jian-jun GAO and KeWei WANG revised the paper.

\section{References}

1 Xia J, Song P, Sun Z, Sawakami T, Jia M, Wang Z. Advances of diagnostic and mechanistic studies of gamma-glutamyl transpeptidase in hepatocellular carcinoma. Drug Discov Ther 2016; 10: 181-7.

2 Sun Z, Zhu Y, Xia J, Sawakami T, Kokudo N, Zhang N. Status of and prospects for cancer vaccines against hepatocellular carcinoma in clinical trials. Biosci Trends 2016; 10: 85-91.

3 Chen L, Wang L, Diao X, Qian W, Fang L, Pang Y, et al. The diagnostic value of contrast-enhanced ultrasound in differentiating small renal carcinoma and angiomyolipoma. Biosci Trends 2015; 9: 252-8.

4 Sun H, Song T. Hepatocellular carcinoma: Advances in diagnostic imaging. Drug Discov Ther 2015; 9: 310-8.

5 Song T. Recent advances in surgical treatment of hepatocellular carcinoma. Drug Discov Ther 2015; 9: 319-30.

6 Zhang L, Jiang $\mathrm{H}, \mathrm{Xu} \mathrm{G}$, Chu N, Xu N, Wen H, et al. iTRAQ-based quantitative proteomic analysis reveals potential early diagnostic markers of clear-cell Renal cell carcinoma. Biosci Trends 2016; 10 : 210-9.

7 Escudier B, Eisen T, Stadler WM, Szczylik C, Oudard S, Siebels M, et al. Sorafenib in advanced clear-cell renal-cell carcinoma. N Engl J Med 2007; 356: 125-34.

8 Llovet JM, Ricci S, Mazzaferro V, Hilgard P, Gane E, Blanc JF, et al. Sorafenib in advanced hepatocellular carcinoma. N Engl J Med 2008; 359: $378-90$.

9 Ge S, Huang D. Systemic therapies for hepatocellular carcinoma. Drug Discov Ther 2015; 9: 352-62.

10 Liu L, Cao Y, Chen C, Zhang X, McNabola A, Wilkie D, et al. Sorafenib blocks the RAF/MEK/ERK pathway, inhibits tumor angiogenesis, and induces tumor cell apoptosis in hepatocellular carcinoma model PLC/ PRF/5. Cancer Res 2006; 66: 11851-8.

11 Ramos-Torres A, Bort A, Morell C, Rodriguez-Henche N, Diaz-Laviada I. The pepper's natural ingredient capsaicin induces autophagy blockage in prostate cancer cells. Oncotarget 2016; 7: 1569-83.

12 FDA Approves New Drug Treatment for Long-Term Pain Relief after Shingles Attacks. http://www.fda.gov/NewsEvents/Newsroom/ PressAnnouncements/ucm191003.htm (accessed January 20, 2017).

13 Jang JJ, Cho KJ, Lee YS, Bae JH. Different modifying responses of capsaicin in a wide-spectrum initiation model of F344 rat. J Korean Med Sci 1991; 6: 31-6.

14 Kaymaz BT, Cetintas VB, Aktan C, Kosova B. MicroRNA-520a-5p displays a therapeutic effect upon chronic myelogenous leukemia cells by targeting STAT3 and enhances the anticarcinogenic role of capsaicin. Tumour Biol 2014; 35: 8733-42.

15 Jin J, Lin G, Huang H, Xu D, Yu H, Ma X, et al. Capsaicin mediates cell cycle arrest and apoptosis in human colon cancer cells via stabilizing and activating p53. Int J Biol Sci 2014; 10: 285-95.

16 Moon DO, Kang CH, Kang SH, Choi YH, Hyun JW, Chang WY, et al. Capsaicin sensitizes TRAIL-induced apoptosis through Sp1-mediated DR5 up-regulation: involvement of $\mathrm{Ca}^{2+}$ influx. Toxicol Appl Pharmacol 2012; 259: 87-95.

17 Jung MY, Kang HJ, Moon A. Capsaicin-induced apoptosis in SK-Hep-1 hepatocarcinoma cells involves $\mathrm{Bcl}-2$ downregulation and caspase-3 activation. Cancer Lett 2001; 165: 139-45.

18 Weber LV, Al-Refae K, Wolk G, Bonatz G, Altmuller J, Becker C, et al. Expression and functionality of TRPV1 in breast cancer cells. Breast Cancer (Dove Med Press) 2016; 8: 243-52.

19 Granato M, Gilardini Montani MS, Filardi M, Faggioni A, Cirone M. Capsaicin triggers immunogenic PEL cell death, stimulates DCs and reverts PEL-induced immune suppression. Oncotarget 2015; 6: 29543-54.

20 Sanchez AM, Malagarie-Cazenave S, Olea N, Vara D, Chiloeches A, Diaz-Laviada I. Apoptosis induced by capsaicin in prostate PC-3 cells involves ceramide accumulation, neutral sphingomyelinase, and JNK activation. Apoptosis 2007; 12: 2013-24.

21 Bhutani M, Pathak AK, Nair AS, Kunnumakkara AB, Guha S, Sethi G, et al. Capsaicin is a novel blocker of constitutive and interleukin-6inducible STAT3 activation. Clin Cancer Res 2007; 13: 3024-32.

22 Zhang Z, Zhou X, Shen H, Wang D, Wang Y. Phosphorylated ERK is a potential predictor of sensitivity to sorafenib when treating hepatocellular carcinoma: evidence from an in vitro study. BMC Med 2009; 7: 41.

23 Abou-Alfa GK, Schwartz L, Ricci S, Amadori D, Santoro A, Figer A, et al. Phase II study of sorafenib in patients with advanced hepatocellular carcinoma. J Clin Oncol 2006; 24: 4293-300.

24 Wilhelm SM, Carter C, Tang L, Wilkie D, McNabola A, Rong H, et al. BAY 43-9006 exhibits broad spectrum oral antitumor activity and targets the RAF/MEK/ERK pathway and receptor tyrosine kinases involved in tumor progression and angiogenesis. Cancer Res 2004; 64: 7099-109.

25 Wei NN, Lv HN, Wu Y, Yang SL, Sun XY, Lai R, et al. Selective activation of nociceptor TRPV1 channel and reversal of inflammatory pain in mice by a novel coumarin derivative muralatin I from Murraya alata. J Biol Chem 2016; 291: 640-51.

26 Tai WT, Cheng AL, Shiau CW, Huang HP, Huang JW, Chen PJ, et al. Signal transducer and activator of transcription 3 is a major kinaseindependent target of sorafenib in hepatocellular carcinoma. J Hepatol 2011; 55: 1041-8.

27 Fattori V, Hohmann MS, Rossaneis AC, Pinho-Ribeiro FA, Verri WA. Capsaicin: current understanding of its mechanisms and therapy of pain and other pre-clinical and clinical uses. Molecules 2016; 21. pii: E844.

28 Yang ZH, Wang XH, Wang HP, Hu LQ, Zheng XM, Li SW. Capsaicin mediates cell death in bladder cancer T24 cells through reactive oxygen species production and mitochondrial depolarization. Urology 2010; 75: 735-41.

29 Mori A, Lehmann S, O'Kelly J, Kumagai T, Desmond JC, Pervan M, et al. Capsaicin, a component of red peppers, inhibits the growth of androgen-independent, p53 mutant prostate cancer cells. Cancer Res 2006; 66: 3222-9.

30 Chow J, Norng M, Zhang J, Chai J. TRPV6 mediates capsaicin-induced apoptosis in gastric cancer cells--Mechanisms behind a possible new "hot" cancer treatment. Biochim Biophys Acta 2007; 1773: 565-76.

31 Lau JK, Brown KC, Dom AM, Witte TR, Thornhill BA, Crabtree CM, et 
al. Capsaicin induces apoptosis in human small cell lung cancer via the TRPV6 receptor and the calpain pathway. Apoptosis 2014; 19: 1190-201.

32 Clark R, Lee SH. Anticancer properties of capsaicin against human cancer. Anticancer Res 2016; 36: 837-43.

33 Irani K, Xia Y, Zweier JL, Sollott SJ, Der CJ, Fearon ER, et al. Mitogenic signaling mediated by oxidants in Ras-transformed fibroblasts. Science 1997; 275: 1649-52.

34 Ramsey MR, Sharpless NE. ROS as a tumour suppressor? Nat Cell
Biol 2006; 8: 1213-5.

35 Renschler MF. The emerging role of reactive oxygen species in cancer therapy. Eur J Cancer 2004; 40: 1934-40.

36 Jiang X, Feng K, Zhang Y, Li Z, Zhou F, Dou H, et al. Sorafenib and DE605, a novel c-Met inhibitor, synergistically suppress hepatocellular carcinoma. Oncotarget 2015; 6: 12340-56.

37 Rollyson WD, Stover CA, Brown KC, Perry HE, Stevenson CD, McNees $\mathrm{CA}$, et al. Bioavailability of capsaicin and its implications for drug delivery. J Control Release 2014; 196: 96-105. 